\title{
Definition of the Map
}

\author{
Miljenko Lapaine ${ }^{\mathrm{a}, *}$, Terje Midtbø ${ }^{\mathrm{b}}$, Georg Gartner ${ }^{\mathrm{c}}$, Temenoujka Bandrova ${ }^{\mathrm{d}}$, Tao Wang ${ }^{\mathrm{e}}$, \\ Jie Shen ${ }^{\mathrm{f}}$ \\ ${ }^{a}$ University of Zagreb, Faculty of Geodesy-mlapaine@geof.hr \\ ${ }^{b}$ Norwegian University of Science and Technology, Dept. of Civil and Environmental Engineering, Geomatics group- \\ terjem@ntnu.no \\ ${ }^{c}$ Technische Universität Wien, Department of Geodesy and Geoinformation-georg.gartner@tuwien.ac.at \\ ${ }^{d}$ University of Architecture, Civil Engineering and Geodesy, Sofia-tbandrova@abv.bg \\ ${ }^{e}$ Geographic Information Science, Capital Normal University, wangt@cnu.edu.cn \\ ${ }^{f}$ Key Laboratory of Virtual Geographic Environment (Nanjing Normal University), Ministry of Education, Nanjing, Jiangsu 210023, \\ China-562972712@qq.com \\ * Corresponding author
}

\begin{abstract}
Cartography has undergone great changes in the last 40 years. Many web platforms and location-based services are offering increasing opportunities, paper maps have been largely supplemented by multimedia and digital maps, and spatial databases. The definition of a map has changed throughout history and the differences in their definitions are presented. This paper aims for new central cartographic definitions, corresponding to contemporary cartographic development after presenting the current situation of the topic. Definitions of cartographic mapping, cartography and cartographer are proposed, as well as a new definition of the map. All they are made on the base of logical analyses including different types of maps from traditional and real to virtual, 3D, animation, and digital.
\end{abstract}

Keywords: map definition, mapping, cartography, cartographer

\section{Introduction and problems observed}

Andrews (1996) published a paper about map definitions. In his historical overview, there are 321 definitions of the term "map". Fortunately, or unfortunately, we can conclude that there are many different map definitions, while no single definition is accepted by all. Over time, definitions of what a map is have changed. Often cartographers make their own definition on the base of their professional background, theoretical and practical knowledge, and research or on the base of use of map.

The mission of the International Cartographic Association (ICA) is to promote the discipline and profession of cartography and GIScience in an international context. Since the map lies at the heart of cartography and GIScience, it is obvious that the ICA must grapple with defining it.

"A map is a symbolized representation of geographic reality, representing selected features or characteristics, resulting from the creative effort of its author's execution of choices, and is designed for use when spatial relationships are of primary relevance." This old definition appears in the ICA Strategic Plan 2003-2011 (ICA 2003), adopted at the 10th General Assembly of the ICA in Barcelona in 1995. So, it is now 25 years old. The main weakness of this definition is the term 'geographical reality', since everybody knows that there are many other elements on maps apart from geographical reality.
Kraak and Fabrikant (2017) give another definition: "A map is a visual representation of an environment". The authors admit in their paper that the map is 'visual' because it usually acts as a visual interface with a virtual or real environment and shows only a selection, thus an abstraction, of the envisaged environment. The map is an interface to an 'environment' which could be a dynamically evolving situation, geographic reality, perceived or cognized reality, virtual, artificial, or a simulated environment, and so on.

The ICA Strategic Plan for 2019-2027 (ICA 2019), includes the following definition, which is rather similar to the one proposed by Kraak and Fabrikant (2017): “A map is an abstract visual representation of the geoenvironment". The third Strategic Plan was presented for approval at the $18^{\text {th }}$ General Assembly of the ICA in Tokyo, Japan, in 2019. During the General Assembly (ICA 2019b) Croatia raised a question about the definitions in the Strategic Plan and presented their concerns. This opinion was seconded by Austria. USA did also point out that the definitions of map and cartography is an important issue and suggested further actions on revisiting the definitions. Belgium suggested a new working group on this issue. The Strategic Plan was accepted with a note that a work proper definition of the "map" should be initiated.

The definition of the map proposed in Tokyo is difficult to understand and accept. We found three problems. The 
first is the meaning of 'abstract' (existing in thought or as an idea, but not having a physical or concrete existence; disassociated from any specific instance; existing as an idea, feeling, or quality, not as a material object). The implication is that a paper map is not a map. The second is the usual meaning of 'visual' (of or relating to seeing or sight). The implication is that a tactile map is not a map. The third is the meaning of 'geo-environment', which is unclear and misleading (for example, see GeoEnvironment - Monitoring, Simulation and Remediation of the Geological Environment, published in 2004). The word 'environment' nowadays is often connected with the concept of environmental protection or sustainability (Malik, Grohmann 2012). In our opinion, spatial relationships, instead of the environment, should be at the core of the definition of the map.

Kraak et al (2021) gave a new definition of the map: an abstracted and authored (often) visual representation of geographic phenomena or processes.

Some remarks to this definition are as follows. The adjective abstracted has many synonyms: miles away, absentminded, distracted, preoccupied, absorbed, engrossed, far away, somewhere else, not there, not with us, in a world of one's own, with one's head, in the clouds, daydreaming, dreamy, inattentive, thoughtful, pensive, lost in thought, deep in thought, immersed in thought, wool-gathering, in a brown study, musing, brooding, absent, distrait, heedless, oblivious. Many of these are not applicable to the definition of a map.

Furthermore, the good definition should not contain anything in parentheses.

Moreover, the adjective visual is not so important in the definition of a map, because the goal of cartography is to model space, and not only to visualize spatial data. A map plays two roles basically: a spatial database (nonvisual) and a communication tool (visual) of spatial information, where the first role is well exemplified nowadays in location-based services and autonomous driving of various mobile vehicles.

Finally, maps do not belong only to geography. Maps are ubiquitous.

The authors of the recent published paper (Gotlib et al, 2021) explore the extended concept of the map in the light of modern geoinformation products. They argue that the development of electronic products based on geoinformation has caused a growing need for the systematization of basic concepts, including defining what a map is. They performed a survey on a group of nearly 900 respondents from a variety of countries. As a result of the survey, the authors of this research are inclined to state that a map should be treated as a specific, ordered information structure and not as a method of presenting spatial data. Unfortunately, they do not propose a new definition of the map.

\section{Definition of the set}

We are going to suggest a new definition of the map by using an idea from mathematics. But we will not use any equations, integrals, or differentials, just something from everyday life.

In everyday life, as in mathematics, we often encounter the notion of sets. Intuitively, a set is a whole composed of elements (objects) that have a common property (Figs $1,2,3)$.

What is a set?

Elementary set theory can be studied informally and intuitively, and so can be taught in primary schools. The basics of set theory have been taught in Croatia since 1975.

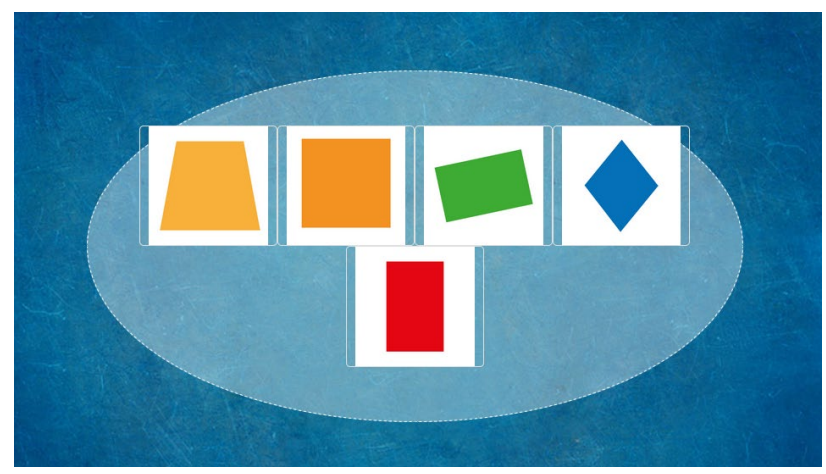

Figure 1. A set of quadrilaterals

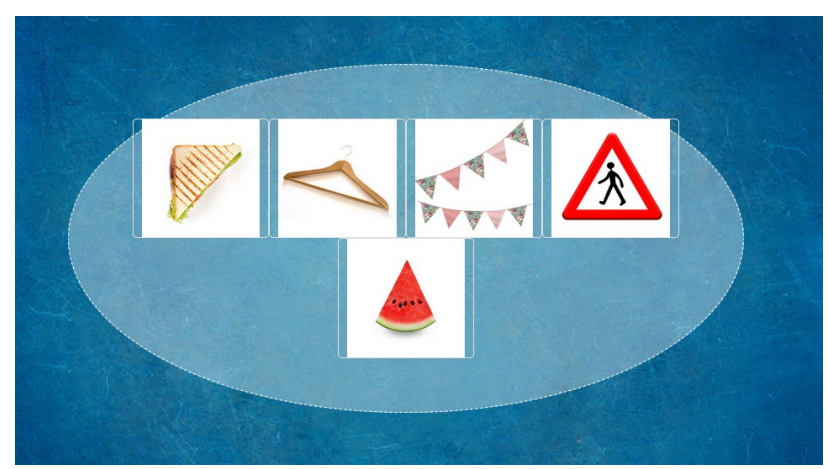

Figure 2. A set of triangular objects

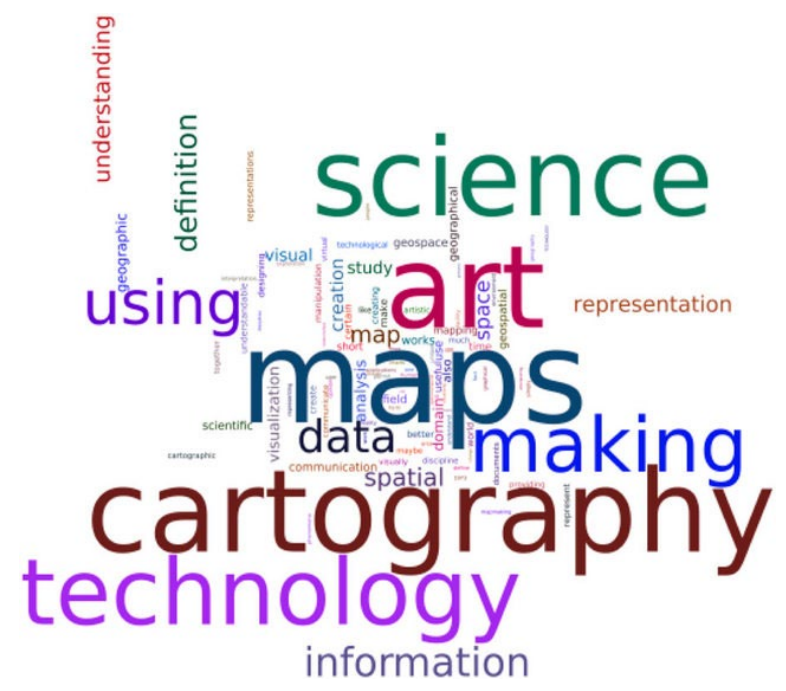

Figure 3. A set of keywords from 'Of maps, cartography and the geography of the International Cartographic Association' by Kraak and Fabrikant (2017) 
The intuitive approach assumes that a set may be formed from a class of objects satisfying a particular defining condition (Figs. 1, 2, 3). Although this may seem a simple idea, sets are one of the most important fundamental concepts in modern mathematics. Set theory, created only at the end of the 19th century, is a ubiquitous part of mathematics teaching today, and is therefore introduced as early as primary school in most countries. Set theory can be understood as the basis on which almost all mathematics can be built.

A set is a collection of objects. The objects that make up a set (also known as the elements or members) can be anything: numbers, people, letters of the alphabet, buildings, rivers, and so on.

\section{Definition of the map}

The definition of the map should include traditional and modern maps, real and virtual maps such as web maps, 3D maps and animation maps, and even globes (Fig. 8a, 8b. We will propose a new definition of the map by using as a background the mathematical concept of mapping from one set to another. Let us start with a simple example. We have two sets (Fig. 4). Ela, Filip, and Ivan are 6th grade students. Ela and Filip like strawberries best, and Ivan loves bananas. How are we going to show that? It could be done as in Fig. 5.
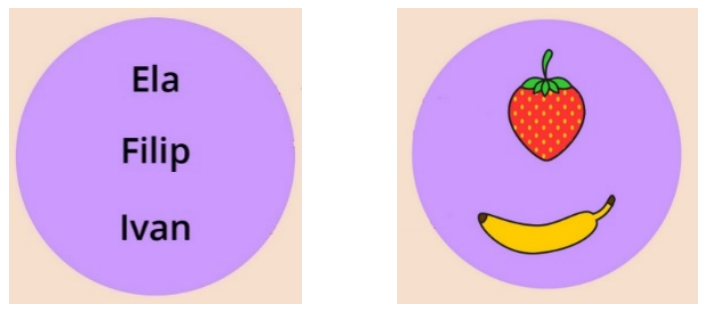

Figure 4. Ela, Filip, and Ivan are students who like strawberries and bananas

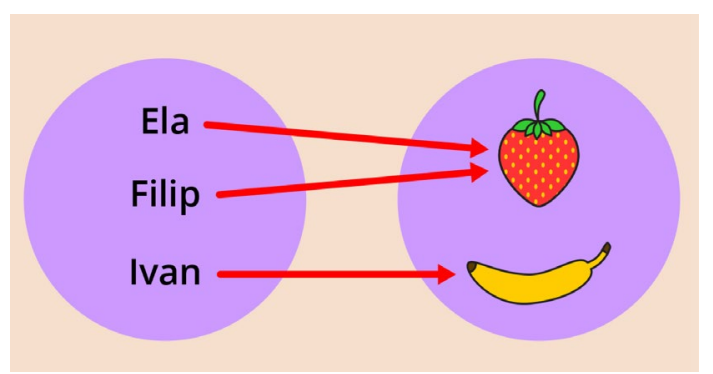

Figure 5. Ela, Filip, and Ivan are 6th grade students. Ela and Filip like strawberries best, and Ivan loves bananas

Figure 6 shows another situation. Ela, Filip, and Ivan are students. Ela goes to school by tram, Filip by tram or bicycle, and Ivan by bicycle or bus.

The elements of the two sets can be linked, connected, joined, associated, put in a relationship, transformed, or mapped in different ways (Fig. 7). We prefer the term mapping due to its neat links with the verb to map and the noun map. The truth is that mapping has many meanings in general (Lapaine 2019). This is why we need a definition of mapping in cartography.

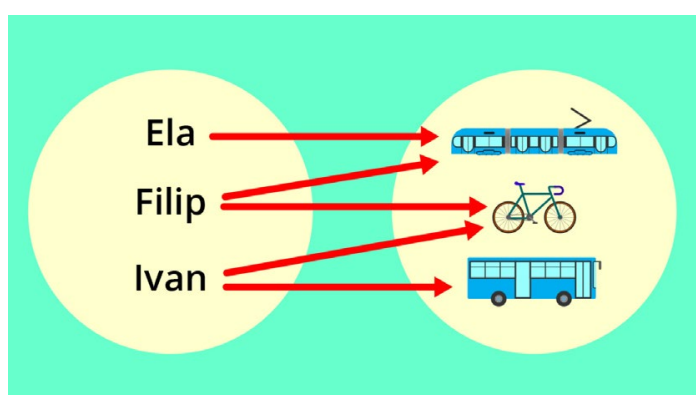

Figure 6. Ela, Filip, and Ivan are 6th grade students. Ela goes to school by tram, Filip by tram or bicycle, and Ivan by bicycle or bus.

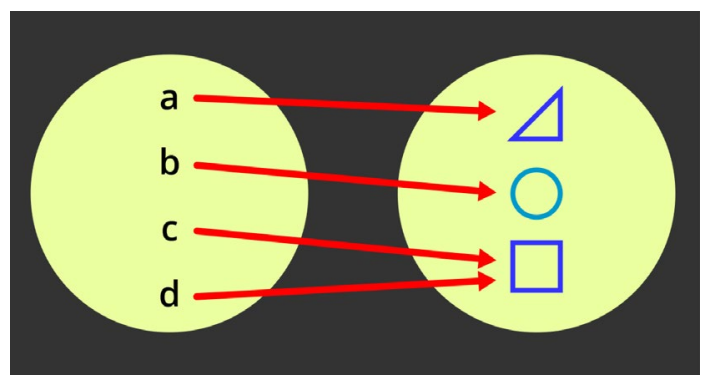

Figure 7. The set on the left (domain) is mapped on the set on the right (codomain)

Mapping is one of the basic terms in mathematics. To recall, two non-empty sets, $X$ and $Y$, are given. By mapping, we understand the rule that each element $x$ from $X$ joins element $y$ from $Y$. So, we write $f: X \rightarrow Y$ (Fig. 9). The element $y$ is the image of $x$, so we write $y=f(x)$. The set $X$ is the domain of mapping $f$, while the set $Y$ is the codomain. Each mapping consists of three parts: the domain, codomain, and mapping rule.

What is a map?

A map is always the result of mapping (Walbert 1997, Weisstein 1997, Rysted 2001). But not any mapping. It should be a special type of mapping (Lapaine 2019) which we will call cartographic mapping. This is a process that associates a set of spatially related data with another set called a map, representation, or model, while preserving spatial arrangements and simplifying detail for specific purposes. Cartographic mapping in German will be kartographische Abbildung, in French application cartographique, in Russian картографическое отображение, in Croatian kartografsko preslikavanje.

The codomain of cartographic mapping is usually a flat surface as a piece of paper or monitor screen. However, it can also be produced of different materials for tactile maps, or it is just imagination, as is the case with mental maps.

Lots of things are represented on maps. The domain of cartographic mapping can be any set of spatial data, for example the surface of the Earth, Moon or Mars, depths of the sea, temperature distribution, population density, economic production, or fictional regions (Fig. 8).

In our opinion, spatial components are the most important part of maps and cartography. By preserving spatial arrangements, we mean preserving topology, that 

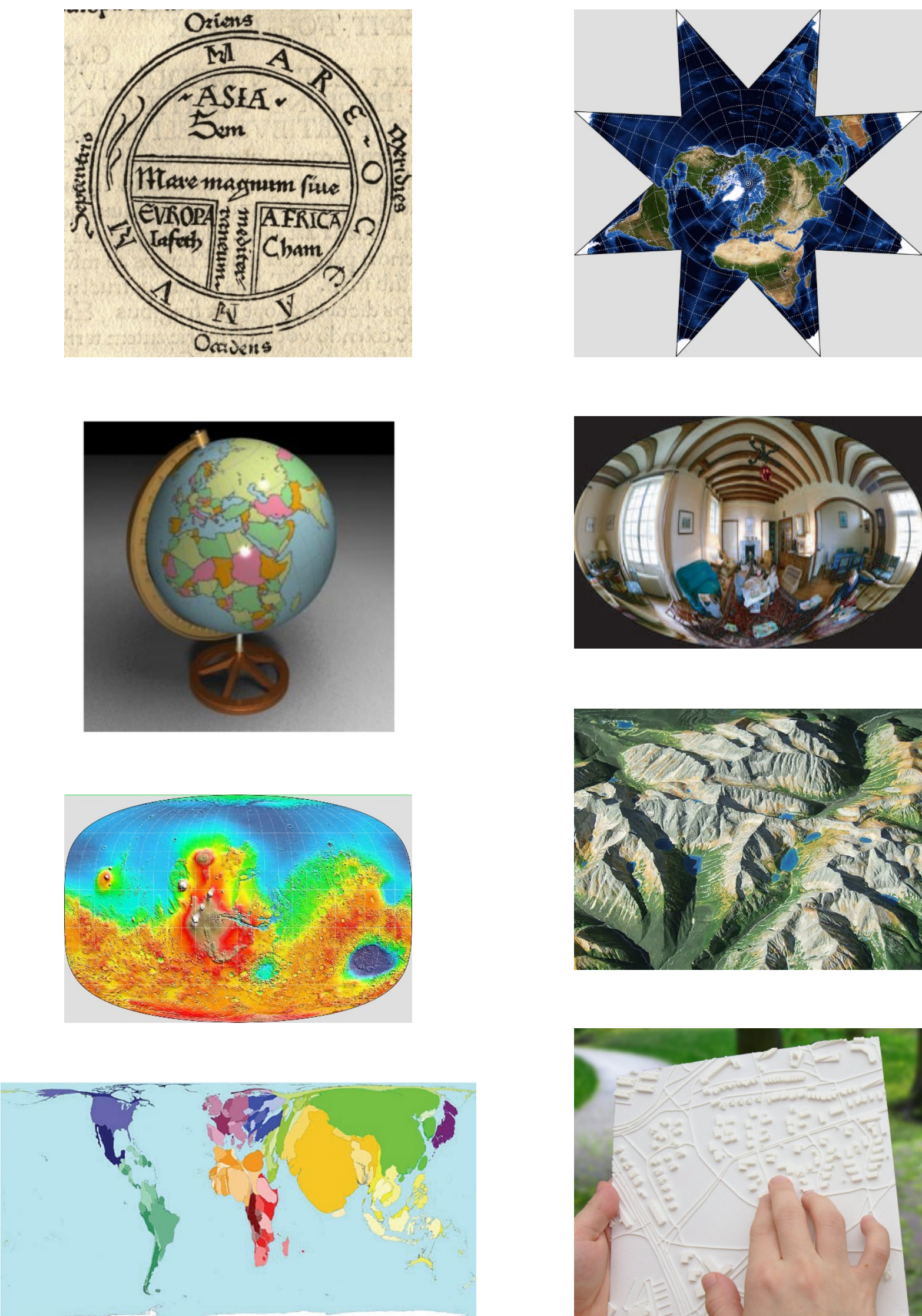

Figure 8. Different types of maps
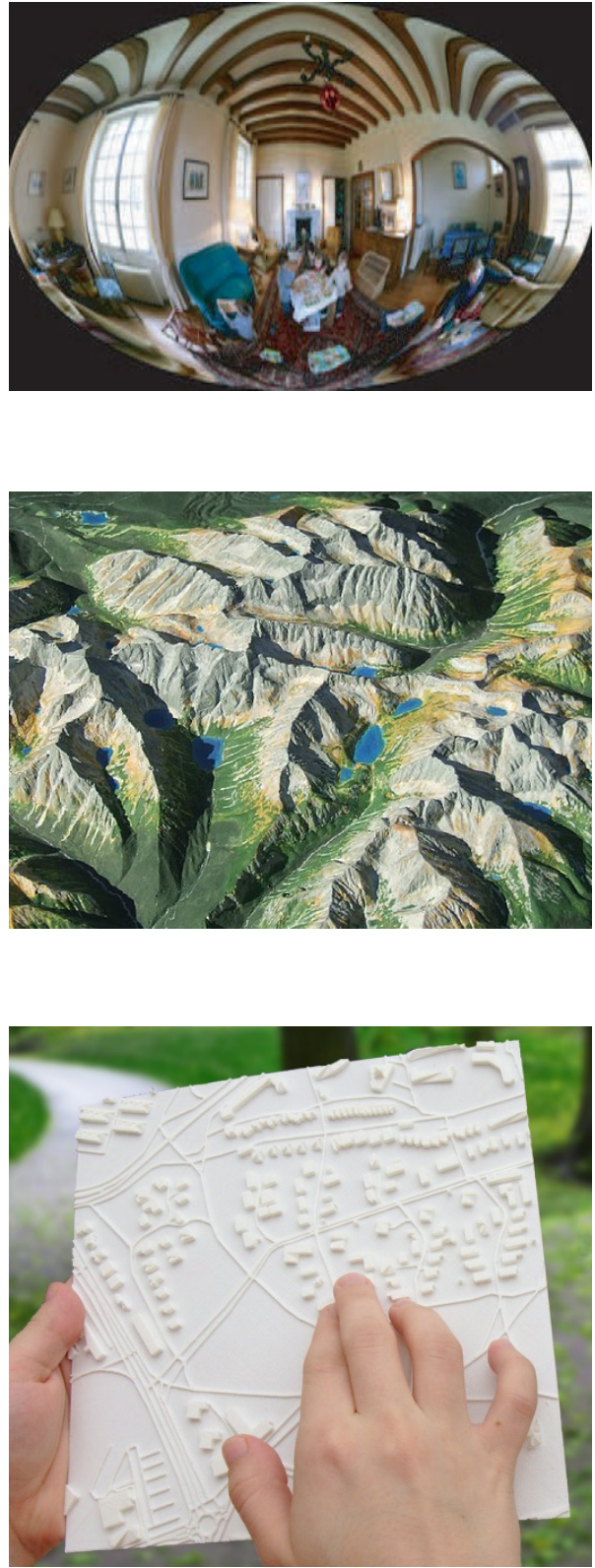


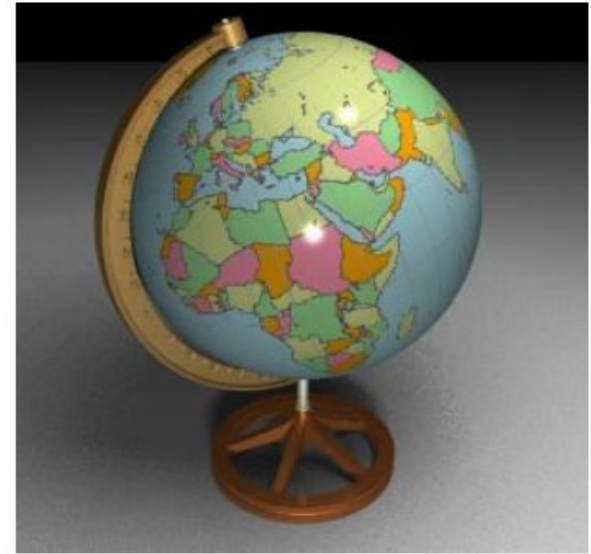

Figure 9. The set on the left (domain) is mapped on the set on the right (codomain)

is, relations such as 'between', 'in front of', behind', 'in', 'outside' and so on. For instance, if a road and a river run parallel in the landscape, then their images should not intersect on a map. Cartographic mapping should be continuous deformation, such as translation, rotation, stretching, twisting, crumpling, and bending, but not tearing or gluing, in general.

However, if we need a definition of the map without introducing mapping as an intermediate step, then we would express it like this. A map is a result of a process that associates a set of spatially related data with their representation, while preserving spatial arrangements and simplifying detail.

Much shorter definition would be:

A map is a generalized representation of spatial relationships.

The ICA Working Group on the Cartographic Body of Knowledge (WG CartoBoK) https://icaci.org/workinggroups/) has in its Terms of Reference the task to review current terms related to cartography and propose new terms and updated definitions to current terms as appropriate. The members of the $\mathrm{WG}$ discussed the definition of a map at sessions held over the past few months (2020-2021). They came to a conclusion that a map is the result of mapping, but the mapping that creates a map always wish to communicate something. So, current WG CartoBoK definition of a map is:

A map is a medium designed for communication of generalized spatial information and relationships.

Note: A map often includes a title, scale, map projection, cartographic network or coordinate grid, legend, source citation, information on the map author, publisher, and date of production. A map is usually produced in visual form and serves for communication using cartographic symbols.

In this last version of the definition the term representation (...or model) is not included. When we are "communicating generalized information" we implicitly talking about a representation/model.
This definition includes traditional and modern maps. There are many different cartographic mappings that result in different kind of maps and communicate different messages (Fig. 8a and 8b).

\section{Definition of cartography and the cartographer}

Regardless of the definition of the map, we think that most people involved in cartography would accept that cartography is the science, technology and art of cartographic mapping and using maps.

We live in an age of the rapid proliferation of computer games. So, it is no wonder that if we type the word cartographer into a search engine, we find 'Ellen' (2020). Who is Ellen, and is she really a cartographer? She is the main character in an action role-playing game. Obviously, Ellen is not a cartographer in the usual sense.

We propose a very simple definition: the cartographer is a person involved in cartography. It can be a mapmaker, scientist, teacher, archivist, librarian, collector of maps or any other person engaged in cartography.

\section{Conclusion}

The technology for producing maps has changed fundamentally in the last 40 years. Nowadays, paper maps are produced exclusively using digital technology. However, they are increasingly being replaced by multimedia or electronic maps. Global cartographic services provide potential applications which were recently unimaginable. Due to all the changes in cartography, we thought it is necessary to produce a new definition of main cartographic terms. Based on the preceding sections, we propose the following new definitions:

Cartographic mapping is mapping a set of spatially related data, while preserving spatial arrangements and simplifying detail.

A map is a medium designed for communication of generalized spatial information and relationships.

Cartography is the science, technology and art of cartographic mapping and using maps.

A cartographer is a person involved in cartography. 


\section{References}

Andrews J H (1996): What Was a Map? The Lexicographers Reply, Cartographica, Vol. 33, No. 4, 111

Ellen (2020): Computer game Ellen the cartographer, https://www.youtube.com/watch?v=HlmBNjomRho (accessed 15 Dec 2020)

Gotlib D, Olszewski R, Gartner G (2021): The Extended Concept of the Map in View of Modern Geoinformation Products. ISPRS Int. J. Geo-Inf. 10, 142. https://doi.org/ 10.3390/ijgi10030142

ICA (2003): Strategic Plan 2003-2011, https://ica.org/ files/documents/reference_docs/ICA_Strategic_Plan_20 03-2011.pdf

ICA (2011): Strategic Plan 2011-2019, https://icaci.org/ files/documents/reference_docs/ICA_Strategic_Plan_20 11-2019.pdf

ICA (2019): Strategic Plan 2019-2027, https://icaci.org/ files/documents/generalassembly2019/22-ica_strategic plan_2019-2027.pdf (online from 1 Dec 2020)

ICA(2019b): Minutes of the $18^{\text {th }}$ General Assembly of the ICA. https://icaci.org/general-assembly-2019 (accessed 17 Mar 2021

Kraak M-J, Fabrikant S I (2017): Of maps, cartography and the geography of the International Cartographic Association, International Journal of Cartography, 2017, Vol. 3, No. S1, 9-31, https://doi.org/10.1080/23729333. 2017.1288535
Kraak M-J, Roth R E, Ricker B, Kagawa A, Le Sourd G (2021): Mapping for a Sustainable World, The United Nations: New York, NY (USA), https://unite.un.org/ news/mapping-sustainable-world

Lapaine M (2019): Mapping in Cartography, Proceedings of the International Cartographic Association, 2, 2019. 29th International Cartographic Conference (ICC 2019), 15-20 July 2019, Tokyo, Japan, str. 1-3. https://doi.org/ 10.5194/ica-proc-2-70-2019

Malik A, Grohmann E (eds. 2012): Environmental Protection Strategies for Sustainable Development, Springer Netherlands, doi 10.1007/978-94-007-1591-2

Martin-Duque J F, Brebbia C A, Godfrey A E, Diaz de Teran J R (2004): Geo-Environment - Monitoring, Simulation and Remediation of the Geological Environment, ISBN 978-1-85312-723-6, Transaction Series WIT Transactions on Ecology and the Environment, Vol. 75, pages 376

Rystedt, B. (2001): New Trends and 558 Developments in Cartography. International Cartographic Association. Presented at CODI-2, Addis Ababa, September 4-7, 2001

Walbert, D. (1997): Map skills and higher-order thinking, http://web.archive.org/web/20170731080509/http://ww w.learnnc.org/lp/editions/mapping/6428 (accessed 8 Aug 2019)

Weisstein, E. W. (1997) "Map" from MathWorld - A Wolfram Web Resource, http://mathworld.wolfram. com/Map.html (accessed 8 Aug 2019) 